Alexander Toepel

Frankfurt am Main atoepel@gmx.net

\title{
EIN BRIEF PAPST JOHANNES‘ XXII. AN DEN KOREANISCHEN KÖNIG*
}

In den von Aloysius Tautu im Rahmen der Quellen zum Codex Iuris Canonicis Orientalis herausgegebenen acta Johannes' ${ }^{\prime}$ XXII. findet sich ein Konvolut von vier Briefen zur Amtseinführung eines neuen Erzbischofs von Khanbaliq, d.i. Beijing, die an den Großkhan der Mongolen, andere mongolische Herrscher, das mongolische Volk und an eine Person namens Socus gerichtet sind. Letzterer wird als rex Corum bezeichnet, was bereits im neunzehnten Jahrhundert auf die Halbinsel Korea bezogen wurde. ${ }^{1}$ Dieser Brief, der schon 1949 von Jean Richard in einer Notiz bemerkt wurde, soll im folgenden zunächst in seinem lateinischen Wortlaut und in deutscher Übersetzung vorgestellt werden, um ihn dann in den Kontext der römisch-katholischen und nestorianischen Mission in Ostasien um die Wende vom 13. zum 14. Jahrhundert einzuordnen. ${ }^{2}$

${ }^{*}$ ) Eine frühere Fassung dieses Beitrags wurde am 21. September 2010 auf dem 31. Deutschen Orientalistentag in Marburg vorgetragen. Das Thema wurde gewählt im Hinblick auf die Ehrung eines Gelehrten, dem es in meisterhafter Weise gelungen ist, in der mediävistischen Forschung Ost und West zu verbinden. Für Rat bei der Übersetzung des lateinischen Textes ist der Autor Herrn Prof. P. Dr. Rainer Berndt SJ, Frankfurt am Main, zu Dank verpflichtet.

(1) Vgl. A. L. TAutu (Hg.), Acta Ioannis XXII. (1317-1334) (Rom: Typis Polyglottis Vaticanis, 1952) (Fontes, ser. 3, vol. 7, tom. 2) 255-258 (Nr. 138), bes. $258 \mathrm{~m}$. Anm. 1 .

(2) Der lateinische Text folgt dem Druck von C. Baronius u.a. (Hg.), Annales Ecclesiastici 24: 1313-1333 (Bar-le-Duc u.a.: Consociatio Sancti Pauli, 1880), 524; vgl. auch Tautu, Acta..., 258 und G. Mollat, Jean XXII (1316-1334). Lettres communes 13 (Paris: De Boccard, 1933) (Lettres communes des Papes d'Avignon), 191 (Nr. 63874). Vgl. dazu J. Richard, Un texte latin du XIVe siècle concernant la Corée, in: Actes du XXI ${ }^{e}$ Congrès International des Orientalistes (Paris: Société Asiatique de Paris, 1949) 349-350. 
Magnifico viro Soco de Chigista ${ }^{3}$ regi Corum Deum diligere et timere. Deo vivo et vero Regi regum et dominantium Domino gratum praestas obsequium cum Christianos sive antiquos sive novellos in fide Christi commorantes in regno tuo benigna humanitate prosequeris et favoris mansuetudine tractas illos; quod quidam laetanter audivimus te fecisse. Unde cum hoc nomini tuo bonam famam adiiciat et honorem tu levans tuae mentis oculos ad divinae omnipotentiam maiestatis circa id benigne continues in posterum quod hucusque laudabiliter effecisti: nam ab hoc in gloria firmabitur tuum solium dicti regni et si credideris in Dominum nostrum Iesum Christum et fueris baptizatus legemque Christianam sine qua nullus salvatur susceperis et impleveris salvus eris.

An den hochwohlgeborenen ${ }^{4}$ Mann Socus von Chigista, König der Koreaner, ${ }^{5}$ Gottesliebe und Gottesfurcht. Dem lebendigen und wahren Gott, König der Könige und der Herrschenden Herr, leistest du erwünschten Gehorsam, indem du dich den alten oder neuen Christen, die in deinem Reich im Glauben Christi ausharren, mit gütiger Menschlichkeit zuwendest und jene nach Gewohnheit der Gunst behandelst; was du, wie wir mit Freude hörten, wirklich tatest. In dieser Hinsicht wird deinem Namen guter Ruf und Ehre hinzugefügt, wenn du die Augen deines Geistes zur Allmacht der göttlichen Majestät erhebst und mit dem, was du bis jetzt lobenswerterweise getan hast, auch in Zukunft gütig fortfährst: Denn dadurch wird deine Königswürde über besagtes Land in Ehre befestigt werden, und wenn du an unseren Herrn Jesus Christus glaubst, getauft wirst und das christliche Gesetz, ohne das niemand zur Erlösung kommt, auf dich nimmst und erfüllst, wirst du erlöst werden.

Nach der Notiz im Druck des Briefes bei Baronius, wo das Schreiben nicht vollständig wiedergegeben wird, fordert der Papst den König außerdem auf, sich den Verkündern des Evangeliums zu unterwerfen und den neuen Erzbischof von Khanbaliq wohlwollend aufzunehmen. Aus der Datumsangabe Avinione kal. Octobris anno XVIII

(3) Geändert aus Secede Chigista nach TAutu, Acta..., 258. Das Original befindet sich in Reg. Aven. 46, f. 138 und Reg. Vat. 106, f. 2v. Vgl. auch J. MulDoon, The Avignon Papacy and the Frontiers of Christendom: The Evidence of Vatican Register 62, Archivum Historiae Pontificiae 17 (1979) 125-195, hier: 173-179.

(4) magnificus nach J. F. Niermeyer u.a., Mediae Latinitatis Lexicon Minus M-Z (Darmstadt: WBG, $\left.{ }^{2} 2002\right)$, s.v. „Freier vornehmer Herkunft”.

(5) Nach Tautu, Acta..., 258, Anm. 1. 
geht hervor, daß der Brief am 1. Oktober 1333 geschrieben wurde, d.h. etwas mehr als ein Jahr vor dem Tod Papst Johannes' XXII. am 4. Dezember 1334. ${ }^{6}$ Johannes war um 1244 als Jacques Duèse in Cahors im Südwesten Frankreichs geboren und 1300 nach dem Studium der Rechte in Montpellier und Orléans zum Bischof von Fréjus ernannt worden. Seit 1310 Bischof von Avignon, wurde er am 5. September 1316 zum Papst gekrönt; er residierte zeitlebens in Avignon. Sein Pontifikat war geprägt durch die Auseinandersetzung mit Ludwig dem Bayern, die oft vergessen läßt, daß es sich bei ihm um einen juristisch versierten Mann mit lebhaftem Interesse an der Mission im asiatischen Raum handelte. ${ }^{7}$

Hauptzweck der vorliegenden Briefsammlung ist die Amtseinführung eines Nachfolgers des 1328 verstorbenen Erzbischofs von Khanbaliq, Johannes von Montecorvino. ${ }^{8}$ Genannt wird ein Mann mit Namen Nikolaus, der aufgrund anderer Quellen als Franziskaner identifiziert werden kann und am 8. September 1333 von Johannes XXII. designiert worden war. Es ist anzunehmen, daß den begleitenden Schreiben des Papstes eine Gesandtschaft aus Khanbaliq vorausgegangen war, die um einen neuen Bischof ersuchen sollte. Nikolaus scheint erst nach dem 23. September 1334 abgereist zu sein und hatte 1336 das Khanat Tschagatai in Zentralasien erreicht. Da im Jahre 1338 eine weitere Gesandtschaft aus Khanbaliq eintraf, um einen neuen Bischof zu erbitten, ist es fraglich, ob Nikolaus dort eingetroffen ist; möglicherweise fiel

(6) Das gleiche gilt für die anderen drei Briefe; vgl. Mollat, Jean XXII..., 191; Tautu, Acta..., 255.

(7) Zur Person Johannes' XXII. vgl. J. Favier, Les Papes d'Avignon (o.O.: Fayard, 2006) 121-126. 504; J. Grohe, J[ohannes] XXII., in: LMA 5, 544-546; M. Hanst, Johannes XXII., in: BBKL 3, 228-233 (ebd. 232-233 Lit. bis 1992); J. HeFt, John XXII and Papal Teaching Authority (Lewiston u.a.: Mellen, 1986) (Texts and Studies in Religion, 27) 1-17; C.A. LüCKERATH, Johannes XXII., in: TRE 17, 109-112 (112 Lit. bis 1988); L. Vones, Johannes XXII., in: LThK 5, 950-951. Zu seinem Pontifikat vgl. K. A. FInK, Von Johann XXII. zu Clemens VI., in: H. Jedin (Hg.), Handbuch der Kirchengeschichte 3: Die mittelalterliche Kirche 2 (Freiburg u.a.: Herder, 1968) 384-402; K. Hausberger, Die Päpste in Avignon, in: M. Greschat (Hg.), Das Papsttum 1: Von den Anfängen bis zu den Päpsten in Avignon (Stuttgart u.a.: Kohlhammer, 1985) (Gestalten der Kirchengeschichte, 11) 258-274.

(8) $\mathrm{Zu}$ Johannes von Montecorvino vgl. die klassische Monographie von A. van Den Wyngaert, Jean de Monte Corvino, O.F.M., premier évêque de Khanbaliq (1247-1328) (Lille: Société Saint-Augustin, 1924). 
er der Pest zum Opfer, die in den Jahren 1338 bis 1339 in Zentralasien wütete. ${ }^{9}$

Die wichtigste und interessanteste Person ist allerdings der Adressat des Briefes, "Socus". Bei ihm muß es sich um den koreanischen König Chung-suk handeln, der von 1313-1330 und 1332-1339 regierte und sich längere Zeit in Khanbaliq aufhielt. ${ }^{10}$ Am 30. Juli 1294 geboren, war Chung-suk seinem Vater Chung-seon, der selbst nur von 12981299 und 1308-1313 regierte, im Alter von 18 Jahren auf den Thron gefolgt, doch scheint Chung-seon die koreanische Politik bis zu seinem eigenen Tode im Jahr 1325 bestimmt zu haben. ${ }^{11}$ Auf sein Ersuchen hin hielt Chung-suk sich 1321-1325, wenn auch anscheinend widerwillig, in Khanbaliq auf und kehrte erst nach dem Tode seines Vaters in die koreanische Hauptstadt Kaegyeong (das heutige Kaeseong in Nordkorea) zurück. Im selben Jahr heiratete er eine mongolische Prinzessin, die in der Folgezeit einen nicht unbeträchtlichen Einfluß an seinem Hof ausübte. ${ }^{12}$ Zwischen 1330 und 1332 legte er die Regierung zugunsten seines Sohnes Chung-hye nieder, der zu dieser Zeit erst 5 Jahre alt war. Für diese Zeit werden ihm auch eine Reihe von Ausschweifungen zur Last gelegt, deren Historizität sich nicht eindeutig erweisen läßt. ${ }^{13}$ Im Jahre 1339 starb Chung-suk im Alter von 44 Jahren. Seine weltan-

(9) Vgl. Muldoon, The Avignon Papacy..., 173-177; J. Richard, La Papauté et les Missions d'Orient au Moyen Âge (XIII ${ }^{e}-X V^{e}$ siècles) (Rom: École Française, 1998) (Collection de l'École Française de Rome, 33) 152-154.

(10) Richard, Un texte latin..., 349. Für einen ersten Überblick vgl. L. Hambis, Notes sur l'histoire de Corée à l'époque mongole, Toung Pao 45 (1957) 151-218, hier: 194-204; C. N. Weems (Hg.), Hulbert's History of Korea 1 (New York: Hillary House Publ., 1962) 232-235. Der Zusatz de Chigista bezieht sich vielleicht auf seine Abkunft von Dschingis Khan, die ein wichtiges Element in der Herrschaftsbegründung späterer türkischer Herrscher war. Diesen Hinweis verdanke ich Frau Dr. Yukiyo Kasai, Berlin.

(11) Vgl. H.-K. Min, Koryo Politics under Mongol Control: Dynastic Continuity During the Period of Royal Absence, International Journal of Korean History 1 (2000) 17-35, hier: 21-35.

(12) Chung-suk war bereits in den Jahren 1316-1319 verheiratet gewesen, hatte zu dieser Zeit jedoch keinerlei politische Bedeutung besessen; vgl. Haмвis, Notes sur l'Histoire..., 196. Zu seiner zweiten Frau vgl. ebd., 197-200 und Min, Koryo Politics..., 27-29.

(13) Homer Hulbert (Weems, Hulbert's History..., 234) spricht von „a series of events that almost baffles description"; Louis Hambis (Notes sur l'histoire..., 195 Anm. 1) nennt dagegen nur eine einzige Quelle, derzufolge Chung-suks Sohn Chung-hye ein "debauché" gewesen sei. 
schaulichen Neigungen lassen sich aus den Quellen nicht eindeutig erkennen, er scheint aber eine gewisse Präferenz für den Konfuzianismus gehabt zu haben. ${ }^{14}$

Welche Verbindung hatte Chung-suk zu Johannes von Montecorvino und warum heißt es, er habe die Christen in seinem Reich beschützt? Die Antwort auf diese Frage ist im Stammbaum des Königs zu suchen. Sein Vater Chung-seon war der Sohn einer Tochter Khublai Khans, die ihrerseits von einer Frau namens Asujin abstammte. ${ }^{15}$ Dieser Name bedeutet „Alanin“, „Frau alanischer Herkunft“, und da die Alanen, ein Volk kaukasischer Provenienz, aus dem sich die Leibwache der Yüan-Kaiser rekrutierte, seit dem 10. Jahrhundert Christen waren, ist anzunehmen, daß Chung-suk über die Verwandten seiner Großmutter Kontakt zu den christlichen Kreisen der Hauptstadt hatte. ${ }^{16}$ Die Alanen in Khanbaliq hatten sich trotz ihrer ursprünglich byzantinischen Ausrichtung der von Johannes von Montecorvino begründeten katholischen Gemeinde zugewandt, woraus sich die in dem Brief des Papstes vorausgesetzte Verbindung Chung-suks zu den Katholiken erklärt.

Unklar bleibt allerdings, ob es unter den in Khanbaliq lebenden Koreanern oder in Korea selbst so viele Christen gab, daß Chung-suk zu Recht als ihr Beschützer hätte bezeichnet werden können. Aus der Form der Bezeichnungen "Socus" und "Cori“ ergibt sich zunächst, daß sie von Personen übermittelt wurden, die wirklich mit Koreanern Kontakt hatten. Die chinesische Aussprache des Namens „Chung-suk“ lautet Zhong-su, was eine Transkription zu "Socus“ verbietet; der Landesname Goryeo lautet in seiner chinesischen Form Gao-li, von Marco Polo (Kap. 80) mit Cauly wiedergegeben, wohingegen Cori die koreanische Fassung abbildet. ${ }^{17}$ Dazu kommt, daß unter der ersten Regent-

(14) Vgl. WeEms, Hulbert's History..., 233.

(15) Vgl. A. Toepel, Christians in Korea at the End of the 13th Century, in: D. W. WINKLER, L. TANG (Hg.), Hidden Treasures and Intercultural Encounters (Wien u.a.: LIT-Verlag, 2009) (Orientalia-Patristica-Oecumenica, 1) 279-289, hier: 285-286 und die dort genannte Literatur, besonders HАмвis, Notes sur l'histoire..., 180.

(16) $\mathrm{Zu}$ den Alanen und ihrer Rolle am mongolischen Hof vgl. W. BARTHOLD, V. Minorsky, Alān, in: H. A. R. Gibb u.a. (Hg.), The Encyclopedia of Islam. New Edition, 1 (Leiden u.a.: Brill, 1960) 354; A. C. Moule, The Minor Friars in China, Journal of the Royal Asiatic Society (1917) 1-36, hier: 20-26.

(17) Da Marco Polo seinen Reisebericht bereits 1299 niederschrieb, stellt der Brief Johannes' XXII. nicht, wie RIchARD, Un texte latin..., 349 schreibt, die 
schaft von Chung-suks Vater Chung-seon (1298-1299) in Korea ein nestorianischer Mongole mit Namen Giwargis als Chef einer mongolischen Behörde installiert wurde, der sich in seiner kurzen Amtszeit mit Sozialreformen befaßte ${ }^{18}$. Chung-seon selbst war in Khanbaliq in neokonfuzianischen Zirkeln tätig, deren ethische Auffassung sich in vielen Punkten mit der christlichen gedeckt haben würde. ${ }^{19}$ All dies weist im Verbund mit der teilweise alanischen Abstammung Chungseons darauf hin, daß Christen in Korea zumindest eine Zeit lang einflußreiche Positionen innehatten.

Daß sein Sohn Chung-suk sich wenigstens zu Beginn seiner Regierung in einer ähnlichen Umgebung befand oder sogar zu Lebzeiten seines Vaters ein bloßes Ausführungsorgan der Politik Chung-seons war, kann begründet vermutet werden. ${ }^{20}$ Der Brief Johannes' XXII. ist ein weiteres Indiz für die Verflochtenheit des koreanischen Herrscherhauses in die alanisch geprägte katholische Gemeinde von Khanbaliq. Im Hinblick auf den Wortlaut des Briefes ist in dieser Hinsicht nicht auszuschließen, daß auch eine Anzahl von Koreanern den christlichen Glauben, wahrscheinlich in seiner katholischen Gestalt, annahmen. Für eine weitere Verbreitung des Christentums in Korea selbst gibt es dagegen keine Anzeichen; das Christentum scheint in Korea um die Wende des 13. zum 14. Jahrhundert eine höfische und gelehrte Angelegenheit geblieben zu sein. Trotzdem sollte seine Bedeutung in jener Zeit nicht unterschätzt werden: Der hier vorgestellte Brief eines Avignoneser Papstes an das Oberhaupt eines am anderen Ende der Welt gelegenen Landes ist das Dokument einer ersten Verständigung und

erste Erwähnung Koreas in einem abendländischen Dokument dar. Der Brief kann aber als ein erster Beleg für einen tatsächlichen Kontakt zwischen Koreanern und Westeuropäern aufgefaßt werden, was vielleicht bedeutender ist, als die Erwähnung des chinesischen Landesnamens bei Marco Polo.

(18) $\mathrm{Zu}$ dieser Person vgl. H. Franke, $\mathrm{Zu}$ einigen christlichen Personennamen in Texten der Yüanzeit, Zeitschrift der deutschen morgenländischen Gesellschaft 148 (1998) 315-322, hier: 321; ToEPeL, Christians in Korea..., 284-285.

(19) Vgl. Toepel, Christians in Korea..., 286 m. Anm. 33; zu den Hintergründen der neokonfuzianischen Ethik vgl. W. Oмmerborn, Philosophische Grundlagen menschenrechtsrelevanten Denkens im Menzius, Zeitschrift der deutschen morgenländischen Gesellschaft 156 (2006) 413-448, bes. 415, 427, Anm. 39, 429, Anm. 53 zur naturrechtlichen Denkweise der Neokonfuzianer und ihrer Bedeutung für die Frage der Menschenwürde.

Vgl. dazu Min, Koryo Politics..., 26-27. 
vielleicht Auseinandersetzung zwischen der christlichen Tradition des Abendlandes und dem konfuzianisch geprägten Denken Ostasiens.

\section{SUMMARY}

Among the correspondence of Pope John XXII there is a letter to a person called Socus, rex Corum. Written in 1333 upon the occasion of investment of a new archbishop in Khanbaliq/Beijing, this document is in all likelihood addressed to the Korean king Chung-suk (reg. 1313-1330, 1332-1339). Although not being a Christian himself, this monarch had ties to Christianity through maternal descent from an Alan princess, who would have been a Byzantine Christian, and through the ties his father Chung-seon (reg. 1298-1299, 1308-1313) had to Nestorian Mongol magistrates. Living mostly in Beijing, Chung-suk seems to have been involved in the same scholarly circles as his father, where he presumably came into contact with Confucian scholars as well as Nestorian, Orthodox and Roman-Catholic Christians. Pope John's letter to this ruler is another piece in the emerging mosaic of East-Western relationships that took place around the turn of the fourteenth century. 